\title{
Effect of poloidal inhomogeneity in plasma parameters on edge anomalous transport
}

\author{
D.Löchel ${ }^{1}$, M.Z.Tokar ${ }^{2}$, M.Hochbruck ${ }^{1}$, D.Reiser ${ }^{2}$ \\ ${ }^{1}$ Mathematisches Institut, Heinrich-Heine-Universität, \\ Universitätsstrasse 1, 40225, Düsseldorf, Germany \\ ${ }^{2}$ Institut für Energieforschung - Plasmaphysik, Forschungszentrum Jülich GmbH, \\ Association FZJ-Euratom, 52425, Jülich, Germany
}

\begin{abstract}
It is demonstrated that anomalous transport at the plasma edge in tokamaks is essentially affected by poloidal inhomogeneities in the plasma temperature and density arising, e.g., by the formation of Multifaceted Asymmetric Radiation from the Edge (MARFE) at the density limit.
\end{abstract}

Ionization of neutrals released from the wall elements or injected by gas puffing through special valves is an important process in fusion plasmas [1]. The generation of charged particles and the loss of electron thermal energy result in transport processes both along and perpendicular to the magnetic flux surfaces and thus determine the densities and temperatures of plasma components. In the low (L) mode of confinement in tokamaks, which is of interest for the present study, the transport across magnetic surfaces is anomalously large compared to the neoclassical transport expected in quiet plasmas without instabilities [2]. Nowadays it is believed that the transport anomaly at the plasma edge is due to the development of drift-Alfv́en (DA) [3] and drift resistive ballooning (DRB) [4] instabilities, which are driven by Coulomb collisions between charged particles and inhomogeneity of the magnetic field in toroidal devices. These modes are usually analyzed under the assumption that the plasma parameters are constant on magnetic surfaces. Such an approach is justified by the fact that transport processes along the magnetic field lines are generally very fast and the plasma parameters remain nearly homogeneous on magnetic surfaces even if the particle source and associated energy loss are strongly localized. Under certain conditions, however, this assumption is not satisfied. For instance, if the density is ramped up to the Greenwald limit [5] and a plasma belt of very high density and low temperature, the so called Multifaceted Asymmetric Radiation from the Edge (MARFE), arises at the high field side (HFS) [6]. This leads to a strong poloidal variation in the plasma parameters. As it has been demonstrated before $[7,8]$ an interplay between DA and DRB driven types of turbulence can be decisive for density limit phenomena.

In this paper, we consider situations where the neutral particle sources are symmetric in the toroidal direction but strongly inhomogeneous in the poloidal one. Under the L-mode conditions, anomalous perpendicular losses of charged particles are assumed to be driven by DA and DRB micro-instabilities. In order to determine radial fluxes the fluid equations for particle and momentum transfer, the Maxwell equations, and the quasi-neutrality condition are linearized with respect to small perturbations of the densities of charged particles, radial and parallel electric currents, and electric and magnetic fields. These equations are reduced to a second order ordinary differential eigenvalue equation for the poloidal variation of the perturbation eigenfunctions, with coefficients essentially dependend on the equilibrium plasma density and temperature. The eigenvalue is related to the complex frequency of the perturbations [9]. In order to find the $2 \pi$-periodic eigenfunctions, the derivatives are discretized by a suitable pseudo spectral method. After discretization, we end up with a cubic matrix eigenvalue problem, whose solution requires an efficient numerical algorithm. For this purpose a variant of the Jacobi-Davidson method [11], in which the convergence is accelerated significantly by combining it with a multilevel approach, has been elaborated [10].

The poloidal dependence of equilibrium plasma parameters is determined from the heat and pressure balance equations integrated over the radial width of the edge region where neutrals are mostly ionized. Conditions for the MARFE formation in the Tokamak Experiment for Technology Oriented Research (TEXTOR) [13] are analyzed and the importance to calculate the characteristics of drift instabilities and anomalous transport self-consistently with the poloidal structure of plasma parameters is elucidated.

We consider small perturbations of the plasma density $\widetilde{n}$, the electrostatic potential $\widetilde{\varphi}$, the electric current density $\widetilde{\mathbf{j}}=\widetilde{j}_{\|} \mathbf{e}_{\|}+\widetilde{j}_{r} \mathbf{e}_{r}+\widetilde{j}_{y} \mathbf{e}_{y}$, the electric and magnetic fields, $\widetilde{\mathbf{E}}=\widetilde{E}_{||} \mathbf{e}_{\|}+\widetilde{E}_{y} \mathbf{e}_{y}$ and $\widetilde{\mathbf{B}}=\widetilde{B}_{r} \mathbf{e}_{r}$, respectively. Here, $\mathbf{e}_{\|}$denotes the unit vector parallel to the unperturbed magnetic field, $\mathbf{e}_{r}$ the unit vector in radial direction, and $\mathbf{e}_{y}$ corresponds to the direction on the magnetic surface and perpendicular 
to the field lines. These perturbations are governed by the following set of linearized transport equations [14-16], describing the particle continuity

$\frac{\partial \widetilde{n}}{\partial t}+\nabla_{\perp} \cdot\left[n\left(\widetilde{\mathbf{V}}_{E}+\widetilde{\mathbf{V}}_{*}+\widetilde{\mathbf{V}}_{p}+\widetilde{\mathbf{V}}_{\pi}\right)\right]=0$

the force balance perpendicular to the magnetic field

$m_{i} n \frac{\partial\left(\tilde{\mathbf{V}}_{E}+\widetilde{\mathbf{V}}_{*}\right)}{\partial t}+T \nabla_{\perp} \tilde{n}=[\widetilde{\mathbf{j}} \times \mathbf{B}]$

and Ohms law

$-m_{e} n \frac{d \widetilde{V}_{e \|}}{d t}+T\left(\partial_{l} \widetilde{n}+\frac{\widetilde{B}_{r}}{B} \partial_{r} n\right)=-e n \widetilde{E}_{\|}+m_{e} \nu_{e} \frac{\widetilde{j}_{\|}}{e}$.

In addition, the quasi-neutrality of the plasma yields

$\nabla \cdot \tilde{\mathbf{j}}=0$

and by Maxwell's equations we have

$\nabla \times \widetilde{\mathbf{B}}=\mu_{0} \widetilde{\mathbf{j}}, \nabla \times \widetilde{\mathbf{E}}=-\frac{\partial \widetilde{\mathbf{B}}}{\partial t}$.

Here the plasma temperature $T$ is assumed to be identical for electrons and ions and to be invariant under perturbations. Only electrostatic contribution is taken into account in the perpendicular component of the electric field, $\widetilde{E}_{y}=-\partial_{y} \widetilde{\varphi}$. The individual contributions $\widetilde{\mathbf{V}}_{E, *, p, \pi}$ to the ion drift velocity induced by perturbations are explained in [16]. The parallel ion velocity is neglected compared to that of the electrons $V_{e, \|}$, and therefore $\widetilde{V}_{e, \|}=\widetilde{j}_{e, \|} /(e n)$. Due to the toroidal geometry the unperturbed magnetic field is a function of the poloidal angle $\vartheta$,

$B=B_{0} /\left(1+r / R_{0} \cos \vartheta\right)$

where $R_{0}$ is the major radius of the last closed magnetic surface and $B_{0}$ denotes the magnetic field at the surface axis. The low aspect ratio approximation, $r / R_{0} \ll 1$, is adopted henceforth.

We consider perturbations of the form

$\widetilde{n}, \widetilde{\varphi}, \widetilde{\mathbf{j}}, \widetilde{\mathbf{E}}, \widetilde{\mathbf{B}} \sim f(l) \exp \left(-i \omega t+i k_{r} r+i k_{y} y\right)$

where $\omega$ denotes the complex frequency and $\mathbf{k}_{\perp}=k_{r} \mathbf{e}_{r}+k_{y} \mathbf{e}_{y}$ is the perpendicular wave vector. The variation of the perturbation envelope function $f$ along the magnetic field, direction $l$, is due to the $\vartheta$-dependence of the equilibrium parameters. Therefore it is assumed that also $f$ changes with $\vartheta$ only and

$\frac{d f}{d l}=\frac{d f}{d \vartheta} \partial_{l} \vartheta=\frac{d f}{d \vartheta} \frac{1}{q R_{0}}$

Here, $q=B_{\varphi} r /\left(B_{\vartheta} R_{0}\right)$ is the safety factor with $B_{\varphi}$ and $B_{\vartheta}$ being the toroidal and poloidal components of the equilibrium magnetic field. From these assumptions and Eqs. (1)-(5), we obtain, see Ref. [9], the following second order ordinary differential eigenvalue equation for the function $f(\vartheta)$ :

$$
\frac{d^{2} f}{d \vartheta^{2}}+S\left[\bar{\omega}(\bar{\omega}+K) \gamma+\frac{4 L_{n}}{R_{0}}(1-2 \bar{\omega} K \gamma) \cos \vartheta\right] f=0
$$

where

$S=\left(\frac{q R_{0}}{L_{n}}\right)^{2} \frac{\bar{\omega}\left(\frac{m_{e}}{m_{i}} K^{2}+\frac{\beta}{2 \gamma}\right)-\frac{\beta K}{\gamma}+i \frac{L_{n}}{\lambda_{e}} \sqrt{\frac{m_{e}}{m_{i}}} K^{2}}{\bar{\omega}\left(1+4 K^{2} \gamma\right)-K}$,

$\bar{\omega}=\omega L_{n} / c_{s}, K=k_{y} \rho_{s}$ with the density $e$-folding length $L_{n}=-d r / d \ln n$, the ion sound speed $c_{s}=$ $\sqrt{T / m_{i}}$, and the ion larmor radius $\rho_{s} ; \lambda_{e}$ is the electron collision length, $m_{e}$ and $m_{i}$ are the electron and ion masses, respectively, and $\beta=\mu_{0} n T / B^{2}$. The effect of magnetic shear $\widehat{s}=d \ln q / d \ln r$ is taken into 
account through the radial wave vector component $k_{r}$. If $k_{y}$ is large enough, we assume that $k_{r}=k_{y}$ (strong turbulence limit), otherwise $k_{r}$ is bounded from below due to Landau damping of perturbations with too large radial wave lengths [16]. In addition this length has to be smaller than the characteristic scale $L_{n}$ for the radial variation of the plasma parameters. Therefore the factor

$\gamma \equiv \frac{k_{y}^{2}+k_{r}^{2}}{2 k_{y}^{2}}=\max \left(1, \frac{1}{2}+2\left(\frac{\pi}{K} \max \left(\frac{\widehat{s} L_{n}}{q R_{0}}, \frac{\rho_{s}}{L_{n}}\right)\right)^{2}\right)$

appeared in the relationships above.

In the simplest case of poloidally homogeneous equilibrium plasma parameters, Eq. (9) is a Mathieu equation with complex coefficients. This case has been analyzed in Ref.[9] under the assumption that the properties of the eigenvalues of this equation are the same as for the Mathieu equation with real coefficients [17]. Here we consider the situation where the factor $S$ varies with $\vartheta$ because of its dependence on the plasma temperature and density through the electron collision length $\lambda_{e} \sim T^{2} / n$. In this case we have a discrete $N$-spectrum of $2 \pi$-periodic eigenfunctions $f_{N}(\vartheta, K)$ for any fixed $K$. In a linear approximation we can only determine the $\vartheta$-dependence of these eigenfunctions but not their absolute value. The latter is estimated in the mixing length limit for the perturbation of electrostatic potential [16]:

$\frac{e \widetilde{\varphi}}{T}=\frac{\operatorname{Im} \omega}{k_{r} \rho_{s} k_{y} c_{s}} f_{N}(\vartheta, K)$

with the normalization $\int_{0}^{2 \pi}\left|f_{N}(\vartheta, K)\right|^{2} \frac{d \vartheta}{2 \pi}=1$.

The density of the charged particle flux produced by perturbations is determined as $\Gamma_{\perp}=\widetilde{n} \widetilde{\mathbf{V}}_{E}^{*}+\widetilde{n}^{*} \widetilde{\mathbf{V}}_{E}$, cf. [16], and, by taking the relation between the density and potential perturbations into account, one gets

$\Gamma_{\perp}=n c_{s}\left(\frac{\rho_{s}}{L_{\perp}}\right)^{2} \sum_{N, K} \frac{(\operatorname{Im} \bar{\omega})^{3}}{K^{2} \gamma\left(1 / 2+K^{2} \gamma\right)|\bar{\omega}|^{2}}\left|f_{N}(\vartheta, K)\right|^{2}$.

For a given $N$, there is normally a broad $K$-range of unstable perturbations since the typical wave length $2 \pi \rho_{s} / K$ in the $y$-direction, is much smaller than the plasma circumference $2 \pi r$. A straightforward summation over all these modes results in a value $\Gamma_{\perp}$ being too large. Therefore it is argued quite often, that the mode with the largest growth rate $\operatorname{Im} \omega$ dominates the transport on the nonlinear stage of instability. Another possibility is to choose the mode providing the largest particle losses, i.e., the largest surface averaged value of $\Gamma_{\perp},\left\langle\Gamma_{\perp}\right\rangle \equiv \int_{0}^{2 \pi} \Gamma_{\perp} \frac{d \vartheta}{2 \pi}$. Our analysis has shown that both approaches give similar results in general.

In order to demonstrate that poloidal inhomogeneity of the plasma parameters is of importance by considering the anomalous transport at the plasma edge, we examine, as an example, the inhomogeneity arising by the MARFE formation provoked by plasma-wall contact at the high field side (HFS) [6, 13]. Neutrals recycle back into the plasma after the recombination of charged particles at the wall and electrons lose their energy by exciting and ionizing neutral particles. The dependence on the plasma parameters of the total energy $E_{i}$ lost in this process per neutral [18] is well approximated by the formula

$E_{i}=\left[30-16.4 \exp \left(-\frac{5 \cdot 10^{19}}{n}\right)\right] \exp \left[\frac{5.45}{T} \exp \left(\frac{n^{0.26}}{1.72 \cdot 10^{5}}\right)\right]$,

where $T$ and $E_{i}$ are measured in $e V$ and $n$ in $10^{20} m^{-3}$. The poloidal dependence of the neutral influx density $J_{n}$ is assumed to be of the following form

$J_{n}=J_{n}^{\max } \exp \left[-\left(\vartheta-\vartheta_{\max }\right)^{2} / \delta_{\max }^{2}\right]$.

In stationary states, $J_{n}$ max is determined by the total balance between influx of neutral particles and outflow of charged particles, $\left\langle J_{n}\right\rangle=\left\langle\Gamma_{\perp}\right\rangle$.

The energy losses affect the plasma not only locally, but, because of heat conduction along the magnetic field, this distortion can penetrate far away from the neutral entrance position. Through the pressure equilibration an inhomogeneity arises also in the plasma density. Before ionization neutrals penetrate 
somewhat radially into the plasma and a two-dimensional treatment of the plasma region in question is most relevant. Because of coupling with the anomalous transport description, see above, this is still too challenging for a numerical realization and will be tackled in the future. Here we use transport equations integrated over the radial width of the edge region where most of the neutral particles are ionized [9]:

$\delta_{\text {edge }} \approx 1 /\left(n \sigma_{*}\right)$.

Here $\sigma_{*}=\sqrt{m_{i} k_{i} k_{c x} / T}$ denotes the characteristic cross-section for the attenuation of neutrals in plasma, $k_{i}$ and $k_{c x}$ the ionization and charge-exchange rate coefficients, respectively, see, e.g., Ref. [8]. In Eq. (15), $n$ and $\sigma_{*}$ are evaluated at the position where neutrals enter the plasma.

The poloidal distribution of the temperature is governed by the heat balance equation

$\frac{\delta_{\text {edge }}}{q^{2} R_{0}^{2}} \frac{d}{d \vartheta}\left(-\kappa_{\|} \frac{d T}{d \vartheta}\right)=q_{\text {core }}-J_{n} E_{i}-3 \Gamma_{\perp} T$,

where $\kappa_{\|}$represents the parallel plasma heat conduction with the dominant contribution from light electrons [14]. The second and third terms on the right hand side (RHS) correspond to the energy losses by ionization of recycling neutrals and to convection of charged particles out of the confined plasma volume. The density of the heat flux transported from the plasma core into the edge region, $q_{\text {core }}$, depends on $\vartheta$ because the distance between adjacent magnetic surfaces and, thus, the radial temperature gradient vary poloidally due to the Shafranov shift $\Delta$ of magnetic surfaces. Thus we have $q_{\text {core }}=g(\vartheta) P_{\text {heat }} / S$, where $P_{\text {heat }}$ is the heating power transported from the plasma core through the total plasma surface area $S$. The rough estimate $g(\vartheta)=1+\Delta_{1} \cos \vartheta$, obtained in [19] for $\Delta_{1} \approx 2 d \Delta / d r \ll 1$, will be used henceforth. The poloidally averaged plasma density $\langle n\rangle$ is prescribed and the poloidal variation $n(\vartheta)$ is governed by the pressure $n(\vartheta) T(\vartheta)$ being constant along the magnetic flux surface.

Computations have been done for the tokamak TEXTOR [13] with a circular cross-section with minor radius $r=0.46 \mathrm{~m}$, major radius $R_{0}=1.75 \mathrm{~m}$, toroidal magnetic field $B=2.25 T$, safety factor at the plasma edge $q=3.5$ and total heating power $P_{\text {heat }}=1.6 \mathrm{MW}$. Discharges where the plasma column was shifted to the HFS, i.e., $\vartheta_{\max }=\pi$ and $\delta_{\max }=0.1$, have been modelled in order to reproduce the MARFE formation at a critical level of $\langle n\rangle$. Figure 1 shows the self-consistently computed variation of the plasma temperature (top) and the plasma density (middle) poloidal profiles with three given averaged densities $\langle n\rangle=5 \cdot 10^{19} \mathrm{~m}^{-3}$ (dash-dotted curve), $6 \cdot 10^{19} \mathrm{~m}^{-3}$ (dashed curve), $7 \cdot 10^{19} \mathrm{~m}^{-3}$ (solid curve). One can see that for $\langle n\rangle=5 \cdot 10^{19} \mathrm{~m}^{-3}$ the plasma parameters are nearly flat. With increasing averaged density the plasma temperature decreases and density increases at the HFS. At a critical value of $\langle n\rangle=6.5 \cdot 10^{19} m^{-3}$ the state with MARFE arises through the development of the recycling instability [9]. The critical value of $\langle n\rangle$ exceeds the experimental one by $20 \%$. This can be explained by the fact that radiation losses from locally sputtered impurities were not taken into account in the present study. Even at a low density there is a slight ballooning structure in the particle loss, with the maximum of $\Gamma_{\perp}$ located at the low field side (LFS), caused by the inhomogeneity of the magnetic field. This structure is becoming more and more pronounced if $\langle n\rangle$ increases, and $\Gamma_{\perp}(L F S) / \Gamma_{\perp}(H F S) \approx 15$ for $\langle n\rangle=7 \cdot 10^{19} m^{-3}$.

Our analysis shows that the poloidal variation of the plasma parameters influences significantly the characteristics of unstable modes and anomalous transport in the state with MARFE. This is demonstrated by comparing the results obtained for $\langle n\rangle=7.4 \cdot 10^{19} \mathrm{~m}^{-3}$ with different shapes of $T(\vartheta)$ and $n(\vartheta)$ profiles. In the first simulation, we consider the temperature and density profiles similar to those given by the solid curves in Fig. 1. In the second case, these profiles are shifted poloidally by $\pi$, which would correspond to a MARFE at the LFS. Finally, in the third calculation the plasma density and temperature are assumed, as in Ref. [9], constant on magnetic surfaces and equal to the poloidally averaged values: $T(\vartheta)=\langle T\rangle$ and $n(\vartheta)=\langle n\rangle$. In Fig. 2 we present the $K$-spectra of the dimensionless growth rate for the three given sets of profiles. Figure 3 shows the anomalous particle fluxes $\Gamma_{\perp}$ computed with the instability characteristics corresponding to the mode with maximum growth rate. One can see that both the absolute level and the profile shape of the particle loss strongly depend on the temperature and density distributions. In particular, the ballooning structure of $\Gamma_{\perp}$ is much more pronounced for the first profile (solid line), with a "normal" MARFE at the HFS. Practically, there are no at all perpendicular losses of charged particles in the MARFE region at the HFS. The charged particles generated here due to the ionization of injected neutrals are transferred away from the MARFE position with the plasma flows along magnetic field lines, and escape through the last closed magnetic surface due to anomalous 
transport processes at some distance from the point $\vartheta=\pi$. As a result one has to expect a pattern of charged particle outflow to the inner wall with a nearly zero minimum value at the HFS. A quantitative assessement of this behavior will be done in future by considering the particle transport in the scrape-off layer outside the last closed magnetic surface. In the case of the plasma parameters independent of $\vartheta$, dashed-dotted curve in Fig.3, the ratio $\Gamma_{\perp}(L F S) / \Gamma_{\perp}(H F S)$ does not exceed 10 and one has to expect a finite perpendicular flux to the inner wall at $\vartheta=\pi$. It seems realistic that such a difference in the flux patterns can be measured by electric probes which usually operate reliably at a very low plasma temperature in the MARFE region [20]. Therefore we hope that the predicted structure of particle losses in the MARFE state can be verified in the future by comparisons with experimental data.

This work has been supported by the Deutsche Forschungsgemeinschaft through the training group GRK1203.

[1] P.C.Stangeby, The plasma Boundary of Magnetic Fusion Device (IoP publishing, Bristol, 2000) 22.

[2] J.Wesson, Tokamaks, third Edition (Claredon Press, Oxford, 2004) 149.

[3] J.D.Callen, Rev. Lett. 39, 1540 (1977).

[4] P.N.Guzdar, J.F.Drake, D.McCarthy, A. B. Hassam, and C. S. Liu, Phys. Fluids B 5, 3712 (1993).

[5] M.Greenwald, Plasma Phys. Nucl. Fusion, 44, R27 (2002).

[6] J.L.Terry, E.S.Marmar, S.M.Wolfe et al., Bul. Am. Phys. Soc. 26, 886(1981).

[7] B.N.Rogers, J.F.Drake and A.Zeiler, Phys. Rev. Lett. 81, 4396 (1998).

[8] M.Z.Tokar, Phys. Rev. Lett. 91, 095001 (2003).

[9] M.Z.Tokar, F.A.Kelly and X.Loozen, Phys. Plasmas, 12, 052510 (2005).

[10] M.Hochbruck, D.Löchel, "A multilevel Jacobi-Davidson method for a polynomial eigenvalue problem arising in plasma physics", Technical Report, Mathematisches Institut, Universität Düsseldorf (2009)

[11] G.L.G.Sleijpen, A.G.L.Booten, D.Fokkema and H.van der Vorst, BIT 36, 595 (1996)

[12] K.Meerbergen and D.Roose, IMA J. of Num. Anal. 16, 297 (1996).

[13] J.Rapp, P.C.de Vries, F.C.Schüller, M.Z. Tokar, W. Biel, R. Jaspers, H.R. Koslowski, A. Kräamer-Flecken, A. Kreter, M. Lehnen, A. Pospieszczyk, D. Reiser, U. Samm, and G. Sergienko, Nucl.Fusion 39, 765 (1999).

[14] S.I.Braginski, in Review of Plasma Physics, edited by M.Leontovich, Vol.1 (Consultants Bureau, New York, 1963) 193.

[15] R.J.Goldston and P.H.Rutherford, Introduction to plasma physics (IoP publishing, Bristol, 2000) 370.

[16] J.Weiland, Collective Modes in Inhomogeneous Plasma (IoP publishing, Bristol, 2000) 9.

[17] Handbook of mathematical functions, Ninth Printing edited by M.Abramowitz and I.A.Stegun (Dover Public., New York, 1970) 722.

[18] R.K.Janev, D.E.Post, W.D.Langer, K.Evans, D.B.Heifetz, and J.C.Weisheit, J. Nucl. Mater. 121, 10 (1984).

[19] M.Z.Tokar, Phys. Plasmas, 8, 2866 (2001).

[20] M. Lehnen, S.S. Abdullaev, S. Brezinsek, M. Clever, J. W. Coenen, K. H. Finken, M. W. Jakubowski, D. Reiser, D. Reiter, U. Samm, D. Schega, O. Schmitz, H. Stoschus, B. Unterberg and the TEXTOR team, Plasma Fusion Res. 3, S1039 (2008).

Published at Physics of Plasmas april 2009

URL: http://link.aip.org/link/?PHP/16/044508

DOI: $10.1063 / 1.3121222$ 

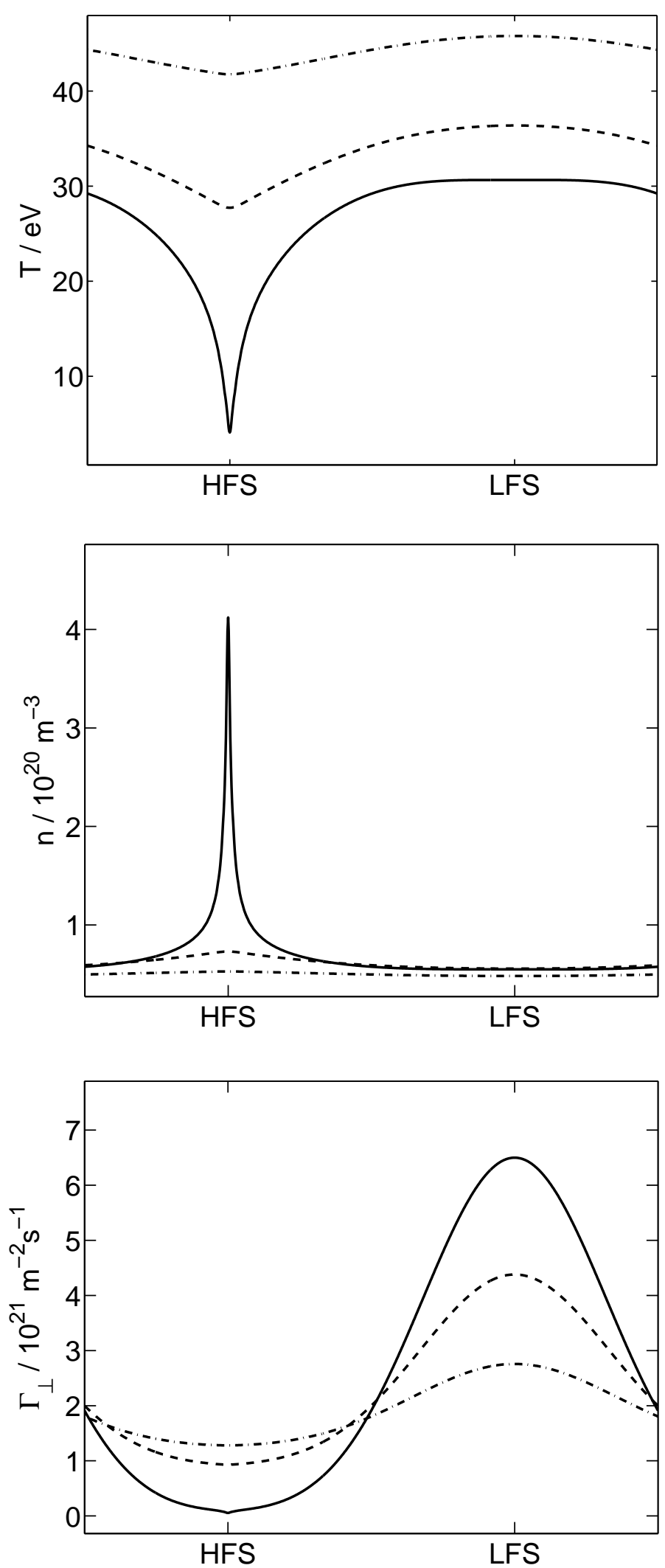

FIG. 1: The poloidal profiles of the plasma temperature (a), density (b) and particle flux density (c) computed for $\langle n\rangle=5 \cdot 10^{19} \mathrm{~m}^{-3}$ (dash-dotted curves), $6 \cdot 10^{19} \mathrm{~m}^{-3}$ (dashed curves) and $7 \cdot 10^{19} \mathrm{~m}^{-3}$ (solid curves). 


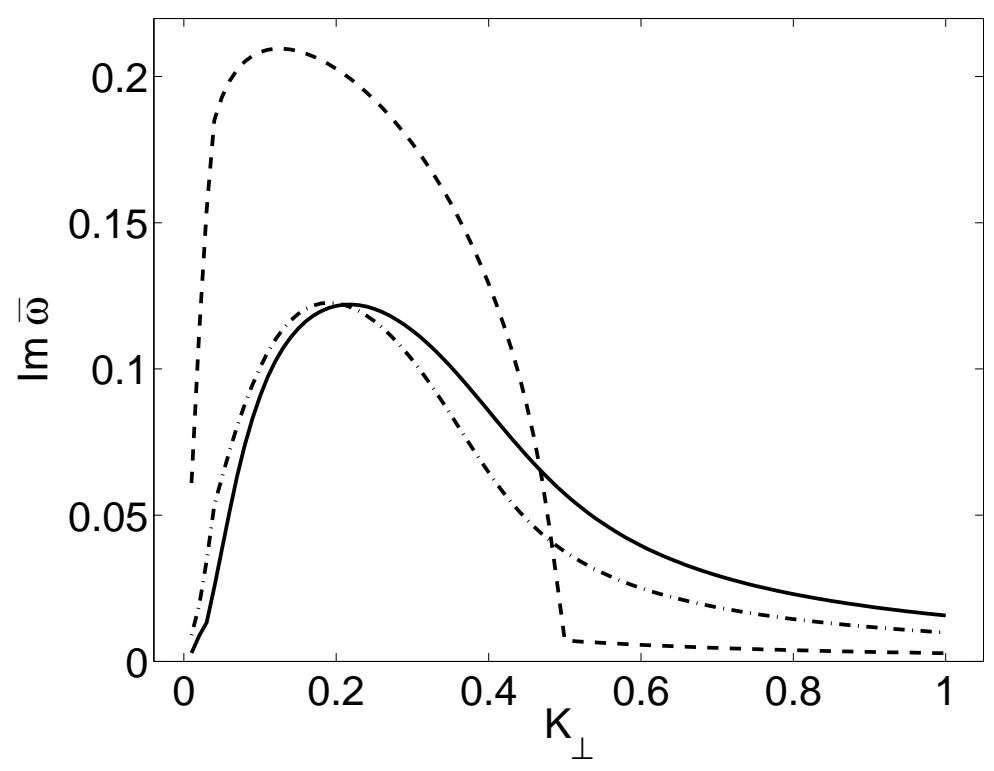

FIG. 2: The $K$-spectra of the dimensionless instability growth rate computed with the plasma parameter profiles calculated self-consistently at $\langle n\rangle=7.4 \cdot 10^{19} \mathrm{~m}^{-3}$ (solid curve), with the same profiles but shifted poloidally by $\pi$ (dashed curve) and with $T(\vartheta)=\langle T\rangle$ and $n(\vartheta)=\langle n\rangle$ (dash-dotted curve).

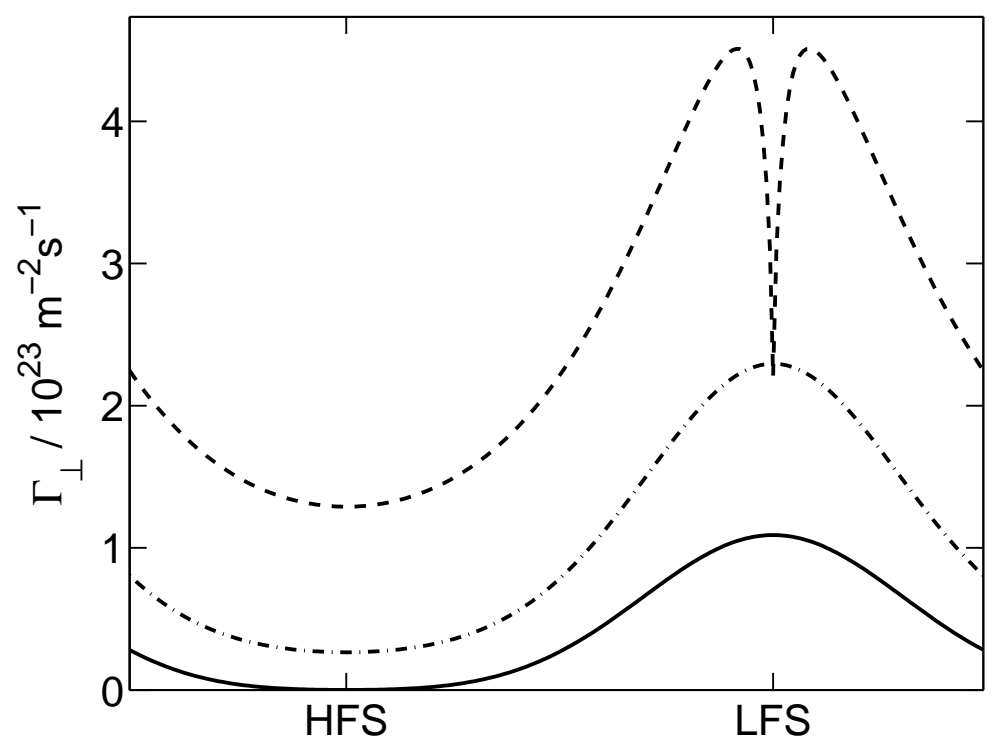

FIG. 3: The poloidal profiles of the charged particle outflux density computed with the plasma parameter profiles calculated self-consistently at $\langle n\rangle=7.4 \cdot 10^{19} \mathrm{~m}^{-3}$ (solid curve), with the same profiles but shifted poloidally by $\pi$ (dashed curve) and with $T(\vartheta)=\langle T\rangle$ and $n(\vartheta)=\langle n\rangle$ (dash-dotted curve). 